Editorial

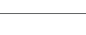

Editorial

\section{Looking forward: our paradigm!}

\section{Editorial}

The last two decades have been exceptional in introducing dramatic advances emanating from genetics and molecular biology as well as the introduction of an unprecedented number of new drugs, particularly for the management of diabetes and osteoporosis, which are transforming the field of endocrinology and metabolism. Numerous recent clinical studies involving common diseases like diabetes, obesity, thyroid disease, osteoporosis, and polycystic ovarian syndrome provide powerful evidence for medical decision-making and treatment. These rapid changes in endocrinology are exciting for professionals and academics in the field in addition to encouraging the need for practicing physicians to continuously update their knowledge base and clinical skills.

While these sources of information are invaluable, the daunting body of data creates an even greater need for synthesis and for highlighting important facts. The facts and evidences must be gone through extensively to find the point of view you want to argue. With a point of view through an editorial piece, issues are given solutions that could be rendered to solve the actual problem at hand. Thus, the preparation of these articles (and reviews) is a special craft that requires the ability to extract core information from the everexpanding knowledgebase. The editors are therefore indebted to our authors, a group of well-recognized health professionals who are masters at providing a comprehensive overview while being able to formulate a topic into a concise and interesting article.

We, as health professionals and patient care-givers, are trying our best to increase our access to medical knowledge and information through web-based journals and databases making this task remarkably efficient in order to accomplish our mission and achieve our goals.

Only through well- designed publication of results in established peer-reviewed journals or scientific forums can new ideas and research findings be disseminated and effectively incorporated into clinical
Volume 2 Issue I - 2015

\author{
Saadi AlJadir \\ Department of Endocrinology, Gulf Medical University, UAE
}

Correspondence: Saadi AlJadir, Department of Endocrinology, Gulf Medical University, P.O. Box 3243, Fujairah, UAE, Email saljadir5I@gmail.com

Received: January 13, 2015 | Published: February 27, 2015

practice, thereby improving physicians' performance and patients' health outcome.

We have ensured that all published reports of research have been reviewed by highly reputed reviewers (e.g. including statistical review where appropriately needed).

Therefore we are intended in our journal to achieve successful medical writing in order to serve as the optimal pathway to advance medical knowledge, promote critical thinking and incite scientific debate by involving many expertise in our publications and maintain the high degree of scientific and professional quality, in addition to considering the continuing progress in different aspects of endocrinology, diabetes and metabolic disorders.

\section{Acknowledgments}

None.

\section{Conflict of interest}

The author declares there is no conflict of interest. 